\title{
AmpliSeq Transcriptome of Laser Captured Neurons from Alzheimer Brain: Comparison of Single Cell Versus Neuron Pools
}

\author{
Wenjun Deng ${ }^{1,2,}$, Changhong Xing ${ }^{1,3},{ }^{*}$, Rob David ${ }^{4}$, Diego Mastroeni ${ }^{5}$, MingMing Ning ${ }^{1,2}$, Eng \\ H. Lo ${ }^{1}$, Paul D. Coleman ${ }^{5, *}$
}

\begin{abstract}
${ }^{1}$ Neuroprotection Research Laboratories, Departments of Radiology and Neurology, Massachusetts General Hospital, Harvard Medical School, Charlestown, MA 02192, USA

${ }^{2}$ Clinical Proteomics Research Center, Department of Neurology, Massachusetts General Hospital, Harvard Medical School, Boston, MA 02114, USA

${ }^{3}$ Department of Pathology, University of Texas Southwestern Medical Center, Dallas, TX 75390, USA

${ }^{4}$ Thermo-Fisher Scientific, Salem, MA 02114, USA

${ }^{5}$ ASU-Banner Neurodegenerative Disease Research Center, Biodesign Institute, Arizona State University, Tempe, AZ 85281, USA
\end{abstract}

[Received December 15, 2018; Revised February 24, 2019; Accepted February 25, 2019]

\begin{abstract}
Alzheimer's disease (AD) is the most common cause of dementia in older adults. However, the pathogenesis of AD remains to be fully understood and clinically effective treatments are lacking. Recent advances in single cell RNA sequencing offers an opportunity to characterize the heterogeneity of cell response and explore the molecular mechanism of complex diseases at a single cell level. Here, we present the application of the Ion AmpliSeq transcriptome approach to profile gene expression in single laser captured neurons as well as pooled 10 and 100 neurons from hippocampal CA1 of AD brains versus matching normal aged brains. Our results demonstrated the high sensitivity and high genome coverage of the AmpliSeq transcriptome in single cell sequencing. In addition to capturing the known changes related to $\mathrm{AD}$, our data confirmed the diversity of neuronal profiles in AD brain, which allow the potential identification of single cell response that might be hidden in population analyses. Notably, we also revealed the extensive inhibition of olfactory signaling and confirmed the reduction of neurotransmitter receptors in AD hippocampus. We conclude that although single neuron data show more variance than data from 10 or 100 pooled neurons, single neuron data can be informative. These findings support the utility of the Ion AmpliSeq method for obtaining and analyzing gene expression data from single defined laser captured neurons.
\end{abstract}

Key words: AmpliSeq transcriptome, single neuron, Alzheimer's disease

Alzheimer's disease (AD) is a degenerative brain disease characterized by progressive cognitive impairment and eventually loss of independence. Over the past several decades, intensive research has revealed unique markers for $\mathrm{AD}$ diagnosis and progression monitoring, which include the formation of beta-amyloid (A $\beta$ ) plaques and neurofibrillary tangles, Tau hyperphosphorylation and apolipoprotein E (APOE) genotypes [1-5]. However,

*Correspondence should be addressed to: Dr. Paul D. Coleman, ASU-Banner Neurodegenerative Disease Research Center, Biodesign Institute, Arizona State University, Tempe, AZ 85281, USA; Email: paul.coleman@asu.edu; Dr. Changhong Xing, Department of Pathology, University of Texas Southwestern Medical Center, Dallas, TX 75390, USA. Email: changhong.xing@ phhs.org; or Dr. Wenjun Deng, Clinical Proteomics Researech Center, Massachusetts General Hospital, Boston, MA 02114, USA. Email: wdeng@mgh.harvard.edu

Copyright: () 2019 Deng W et al. This is an open-access article distributed under the terms of the Creative Commons Attribution License, which permits unrestricted use, distribution, and reproduction in any medium, provided the original author and source are credited. 
since the brain's cognitive function involves the coordinated action of diverse sets of differentiated cell populations [6], the presentation and progression of $\mathrm{AD}$ demonstrates substantial variations in cognitive profile, onset age, and decline rate, and the molecular mechanisms leading to cognitive decline in neurodegeneration remain to be fully defined [7-11]. Therefore, understanding the differential gene expression among single neurons instead of profiling the average expression pattern from pooled samples in complex brain tissue may help to dissect the heterogeneous neural alterations in neurodegeneration and expand our knowledge on AD pathogenesis [12-14].

The possibility of obtaining expression profile from single neurons has been demonstrated since early 1990 s $[15,16]$. With the advances in RNA sequencing technology, it now becomes possible to precisely profile the genome of thousands of cells in a single assay. Recently, Thermo-Fisher Scientific developed an accurate and sensitive RNA sequencing technology, the Ion AmpliSeq transcriptome approach, which is capable of simultaneously amplifying and sequencing over 20,000 pre-defined genes with as little as 10 ng RNA input [17, $18]$. With the advantage of targeted nature and small amplicon size $(\sim 150 \mathrm{bp})$, the turnaround time of Ion AmpliSeq technology is shorter than traditional whole transcriptome RNA sequencing and the number of raw reads assigned to each gene is smaller, which may facilitate experiment operation and data management, particularly when dealing with large dataset. However, up to now, the feasibility of AmpliSeq technology in single cell sequencing has never been evaluated, especially in terms of potentially resolving the heterogeneity of responses in the central nervous system. Here, we present the application of Ion AmpliSeq technology to the determination of expression profiles of single laser captured neurons in Alzheimer's disease (AD) brain versus control neurons from normal aged brain. We focused on the CA1 region of the hippocampus because this is a brain area critical for learning and memory and one that demonstrates rapid neuron loss early on in $A D$ progression. We also compared single neuron profiles to the gene expression obtained from the same tissue sections by concatenating 10 and 100 neurons per reaction. Our results confirmed the potential of the Ion AmpliSeq approach in profiling the whole transcriptome of single neuron and capturing cell-to-cell variation in gene expression. We also provided evidence that single cell sequencing can be more informative in potentially revealing heterogenous $\mathrm{AD}$ related neural alterations, which may be hidden when profiling average gene responses in pooled cell populations.

\section{MATERIALS AND METHODS}

\section{Human subjects}

Frozen unfixed tissue containing Samples of human CA1 hippocampus were secured from one AD (Braak IV) and one ND case (Braak III). Subjects were obtained at autopsy at the Banner Sun Health Research Institute Tissue Bank (BSHRI) in accordance with local IRB approval. BSHRI is a NIA AD Center Brain Bank that features extremely short postmortem intervals (PMIs) (mean of 2.8 hours in over 20 years of operation $[19,20]$, and very high-quality RNA (RIN of 8.5), as well as the detailed annotation required of NIA AD Centers. Cognitive status of all cases was evaluated antemortem by board-certified neurologists, and postmortem examination by a board-certified neuropathologist resulting in a consensus diagnosis using standard NIH AD Center criteria for $\mathrm{AD}$ or neurologically normal, nondemented elderly control. The AD and ND cases were well matched for age (AD: 71 years; ND: 71 years), gender (2 males), postmortem interval (PMI) (AD: 2.1 hours; ND: 2.2 hours) and ApoE genotype E3/E3. RIN values for $\mathrm{AD}$ subject was 7.7 and $\mathrm{ND}$ subject 7.9.

\section{Laser capture of pyramidal neurons}

Frozen brain sections were cut at $15 \mathrm{um}$, stained with $1 \%$ neutral red (Fisher Scientific) and mounted onto PEN slides required for laser capture microdissection. Immediately after staining sections were dipped in $100 \%$ ethanol and loaded onto a Leica AS-LMD laser capture microscope. Pyramidal neurons were identified by their characteristic size, shape, and location in CA1 of the hippocampus. Single neurons were cut out by laser capture under 20X objective. Neurons were dropped into inverted microcentrifuge caps containing proprietary buffer from ThermoFisher. Three single neurons were cut from $\mathrm{AD}$ brain and each one loaded into one of three microcentrifuge caps. Two single neurons from control brain were obtained similarly. The laser capture process was repeated to form caps with 10 neurons in triplicate from $\mathrm{AD}$ and control brain as well as one sample of 100 neurons from $\mathrm{AD}$ and another from control brain.

\section{Ion AmpliSeq ${ }^{\mathrm{TM}}$ Transcriptome Human Gene Expression}

Libraries for RNA sequencing were prepared using AmpliSeq Library kit as previously described [17]. Briefly, total RNA was reverse transcribed into cDNA. Targeted genes were amplified with the Ion AmpliSeq human transcriptome panel, which contains a pool of oligonucleotide primer pairs, each pair designed to amplify a specified genome region. The panel designed for this study targeted 18,574 coding genes and 2,228 non- 
coding genes based on UCSC hg19 annotation. The panel contained 20,802 amplicons (41,604 primers), approximately 150 bases long, in a single pool. Only one amplicon was designed for each gene which means the design is on the common exon shared by multiple isoforms. Following PCR amplification, the amplicons were partially digested with FuPa enzyme and ligated with sample specific barcoded adapters. After removing remaining primers and other residual reaction components, purified amplicon libraries from each sample were combined and were sequenced on Ion Proton $^{\mathrm{TM}}$ sequencer (Thermo-Fisher), with HiQ sequencing chemistry.

Table 1. Summary of AD neuron transcriptome.

\begin{tabular}{lcccc}
\hline Samples & Mapped reads & On target & Targets detected & Genes Identified \\
\hline Single neuron_1 & $5,416,542$ & $90.65 \%$ & $61.51 \%$ & 15,094 \\
Single neuron_2 & $5,871,995$ & $81.56 \%$ & $64.82 \%$ & 15,586 \\
Single neuron_3 & $5,748,512$ & $67.48 \%$ & $64.16 \%$ & 15,582 \\
10 neurons_1 & $6,153,109$ & $83.52 \%$ & $66.98 \%$ & 16,375 \\
10 neurons_2 & $5,793,326$ & $83.08 \%$ & $65.61 \%$ & 16,075 \\
10 neurons_3 & $5,596,528$ & $84.35 \%$ & $65.69 \%$ & 16,662 \\
100 neurons & $4,239,544$ & $89.57 \%$ & $64.90 \%$ & 16,764 \\
\hline
\end{tabular}

\section{Data analysis}

AmpliSeq sequencing data were analyzed using the Ion Torrent Mapping Alignment Program (TMAP) and were normalized using reads per million (RPM). Principal component analysis was performed using $\mathrm{R}$ program. Genes with >2-fold change of mean RPM values between $\mathrm{AD}$ and control neurons were considered as significantly changed and their biological functions were annotated by David Bioinformatics Resources [21]. The significance of the differential expression between AD and control brain was evaluated by Student's t test followed by multiple testing correction with the false discovery rate (FDR) method. FDR greater than 0.05 was considered as statistically significant.

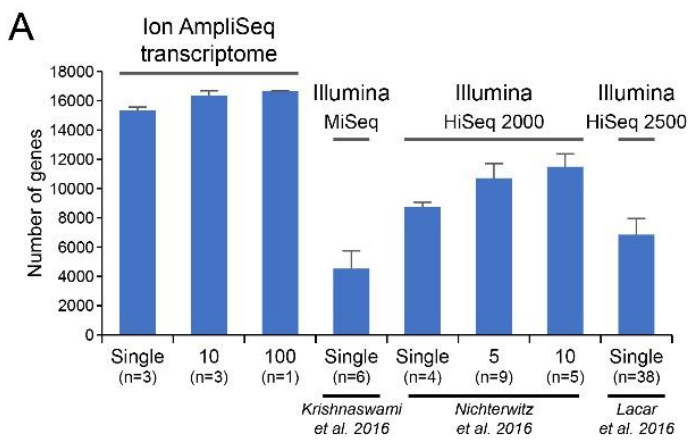

B


Figure 1. Evaluation of AmpliSeq transcriptome sequencing results. (A) Comparison of gene identifications between Ion AmpliSeq technology and other RNA sequencing platform. (B) The distribution of gene abundance in single neuron and pooled 10 and 100 neurons (left) as well as in single neurons with different loading amount (right). (C) Pearson correlation of gene expression between technical replicates. (D) Comparison of gene expression quantified by AmpliSeq with the hippocampus neuron transcriptome measured in other species using different technologies. 

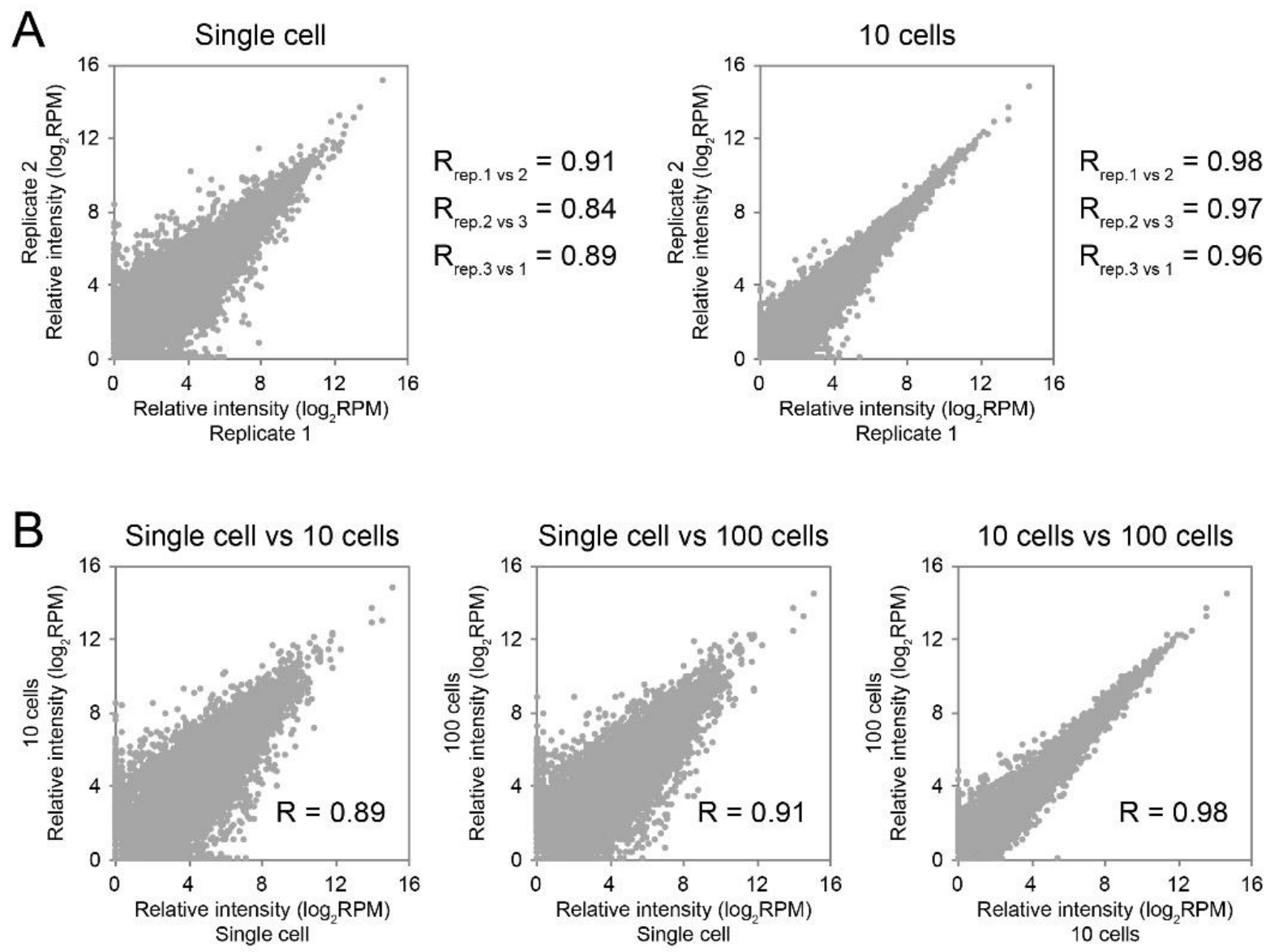

C Principal component analysis

D Gene expression variation within replicates

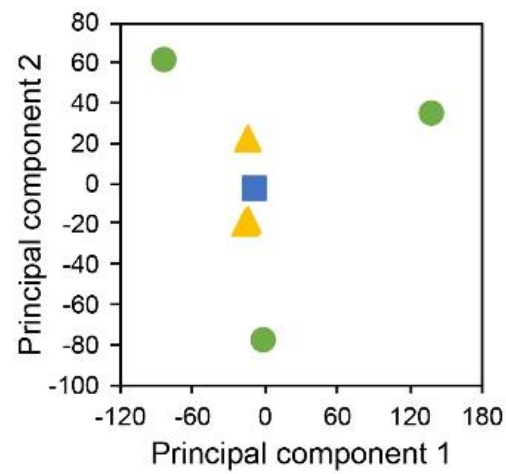

Single cell

10 cells

100 cells

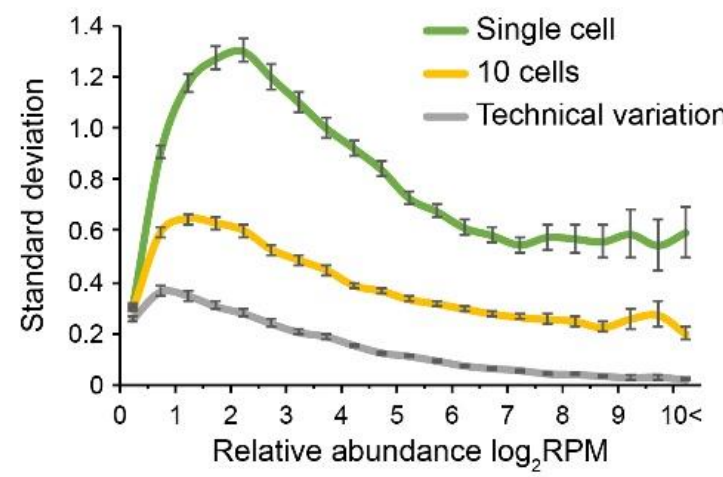

Figure 2. Gene expression diversity in different neuron sets. Pearson correlation of gene expression between replicate experiments (A) and across different neuron sets (B). (C) Principal component analysis of gene expression profiles in different neuron sets. (D) Standard deviation of gene expression within replicates binned according to gene expression levels (mean $\pm 95 \% \mathrm{CI}$ ).

\section{RESULTS}

\section{Evaluation of AmpliSeq neuron transcriptome in AD}

With the AmpliSeq technology, an average of 5.7 million reads were obtained for each single neuron. 67 91\% of these reads (3.9 4.9 million) were on target and led to the identification of $>15,000$ genes in every single neuron, which was comparable to the average 16,371 genes identified in the 10-neuron pool and 16,764 genes in the 100-neuron pool (Table 1 and Table 2). As compared with other RNA sequencing studies, which used different 
platform (Illumina MiSeq and HiSeq) to profile single neuron or neuron pools at a similar scale to our study [2224], Ion AmpliSeq technology largely increased the gene identification coverage, even at single cell resolution (Fig. $1 \mathrm{~A}$ and Table 2). Moreover, similar abundance distribution was found for the genes identified in single neurons as well as in pooled 10 or 100 neurons (Fig. 1B). This remained robust even with different sample loading (5 $\mu \mathrm{l}$ vs. $10 \mu \mathrm{l}-$ Fig. 1B). All these results may support the reliability of the single cell data and also suggest sufficient sensitivity and feasibility of AmpliSeq for single cell sequencing.
In addition, a high gene expression correlation was observed between technical replicates, indicating the reproducibility of AmpliSeq technology (0.999) (Fig. 1C). Significantly correlated gene expression can also be identified when comparing our data with the hippocampus CA1 neuron transcriptome measured in other species (human, mouse, rat) using different technologies (Microarray, Illumina RNA sequencing, Ion Torrent Proton) [25-27], which further confirmed the quantification accuracy and robustness of the AmpliSeq method (Spearman rank correlation: 0.592, 0.538, 0.589, 0.583; $\mathrm{p}<0.001$ ) (Fig. 1D).

Table 2. Comparison with other single neuron sequencing studies.

\begin{tabular}{|c|c|c|c|c|c|c|c|}
\hline \multirow{2}{*}{ Platform } & \multirow{2}{*}{ Neuron origin } & \multirow{2}{*}{$\begin{array}{l}\text { Neuron } \\
\text { sets }\end{array}$} & \multirow{2}{*}{ Replicates } & \multicolumn{4}{|c|}{ Identified genes } \\
\hline & & & & Minimum & Maximum & Average & SD \\
\hline \multirow{3}{*}{$\begin{array}{l}\text { ThermoFisher Ion } \\
\text { AmpliSeq transcriptome }\end{array}$} & \multirow{3}{*}{$\begin{array}{l}\text { Human } \\
\text { hippocampus CA1 }\end{array}$} & Single cell & 3 & 15,094 & 15,586 & 15,421 & 283 \\
\hline & & 10 cells & 3 & 16,075 & 16,662 & 16,371 & 294 \\
\hline & & 100 cells & 1 & N/A & N/A & 16,764 & N/A \\
\hline $\begin{array}{l}\text { Illumina MiSeq } \\
\text { Krishnaswami et al. } 2016\end{array}$ & Human brain & Single cell & 6 & 3,385 & 6,267 & 4,567 & 1,209 \\
\hline \multirow{3}{*}{$\begin{array}{l}\text { Illumina HiSeq } 2000 \\
\text { Nichterwitz et al. } 2016\end{array}$} & \multirow{3}{*}{$\begin{array}{l}\text { Human } \\
\text { motor neuron }\end{array}$} & Single cell & 4 & 8,455 & 9,208 & 8,795 & 310 \\
\hline & & 5 cells & 9 & 8,687 & 12,237 & 10,772 & 1,040 \\
\hline & & 10 cells & 5 & 10,605 & 12,500 & 11,482 & 921 \\
\hline $\begin{array}{l}\text { Illumina HiSeq } 2500 \\
\text { Lacar et al. } 2016\end{array}$ & $\begin{array}{l}\text { Mouse } \\
\text { dentate granule cell }\end{array}$ & Single cell & 38 & 4,553 & 8,769 & 6,843 & 1,135 \\
\hline
\end{tabular}

\section{More diverse gene expression profile in single neurons than in pooled neurons}

We then compared the gene expression profiles across different neuron sets. As suggested by the relatively low correlation between replicates $(0.91,0.84,0.89)$, single neurons demonstrated diverse gene expression patterns (Fig. 2A). This diversity could be reduced by pooling neurons into sets of 10 or 100 , which could be evidenced by the increased correlation of gene expression between replicates of pooled 10 neurons $(0.96,0.97,0.98)$ (Fig. $2 \mathrm{~A})$ as well as between 10 neurons vs. 100 neurons (0.98) (Fig. 2B). The reduced diversity from pooled neurons is further indicated by principal component analysis in which 10-cell and 100-cell pools were concentrated together, while larger distance was found between single neurons (Fig. 2C). Moreover, single neurons demonstrated much higher gene expression variation than the neuron pools and the technical replicates throughout the whole genome range (Fig. 2D). And many neuronal markers, which were used to discriminate different neuron subtypes $[28,29]$, were more differentially expressed in single neurons than the neuron pools (Fig. 3). We therefore concluded that the neuronal composition is highly heterogeneous in the hippocampus of AD patient and Ion AmpliSeq technology is capable of capturing these diversities and can be used to investigate AD-related alterations at a single cell level.

\section{Neuron transcriptome in $A D$ versus Control}

Although single neurons exhibited distinct gene expression patterns, more dramatic differences were observed between samples from AD versus control brains. As shown in principal component analysis (Fig. 4A), neurons from $\mathrm{AD}$ patients and controls can be easily separated. Gene expression changes in AD exhibit a significant positive correlation between single neurons and neuron pools of 10 or 100 neurons (Fig. 4C). Moreover, using a cutoff of $>2$-fold change, around $45 \%$ of the changes in $\mathrm{AD}$ were shared across different neuron sets (Fig. 4D). The expression of APP (amyloid precursor protein) and APPBP2 (APP binding protein 2), which are well characterized genes related to $\mathrm{AD}$, were both significantly up-regulated in single, as well as pooled, AD neurons compared to neurons from control brain (Fig. 4B). All these results suggested that the major changes 
related to $\mathrm{AD}$ are conserved in single hippocampal neurons.

Of note and as expected, single neuron data defined a distinct $\mathrm{AD}$ space versus a distinct control space in the principal component analysis, which reflects the detectable population effects of $\mathrm{AD}$ in spite of diverse expression patterns of single neurons (Fig. 4A). When the diversity of single neurons is eliminated by pooling, the expression correlation between data from 10 vs 100 neurons (0.78) is higher that correlations for single neurons (0.62 and 0.60) (Fig. 4C).


Figure 3. The expression diversity of neuronal markers in different neuron sets. (A) The relative expression of neuron markers across the different neuron sets. (B) Examples of neuron marker expression in single neurons and pooled 10 and 100 neurons.

\section{Functional alterations in single AD neuron and pooled neuron sets}

We then evaluated the functional alterations in $\mathrm{AD}$ with significantly changed genes in single neurons and neuron pools (> 2-fold change). As shown in Figure 5A, many of these changes are known to be affected in AD. Thus, we see involvement of cell death, metabolic processes, mitochondria, synapses, etc. Apoptosis was consistently upregulated in single AD neurons as well as in pooled 10 and 100 neurons with elevated expression for most of the genes involved in apoptosis (Fig. 5B). Notably, proapoptotic genes, endonuclease $\mathrm{G}, \mathrm{Bcl} 2$ like 1 and cytochrome $\mathrm{C}$ were all significantly up-regulated, suggesting enhanced neuron death in AD brain (Fig. 5C).
In contrast, sensory response and G-protein receptor signaling was extensively inhibited in single and pooled AD neurons (Fig. 5A). Moreover, the metabolic processes and synaptic signaling were increased in pooled AD neurons but were less affected in single neurons, suggesting that the small number of single neurons sampled were closer to dying with reduced metabolism and synaptic activity (Fig. 5A). On the other hand, the upregulation of oligodendrocyte development, glial cell development and oxidative stress response pathways was only found in single neurons, but not in pooled neurons (Fig. 5A). As a whole, these data suggested that single cell sequencing with the Ion AmpliSeq system can not only produce consistent data with pooled cell population, but also uncover single neuron responses that are hidden in pooled neuron data sets. 
A

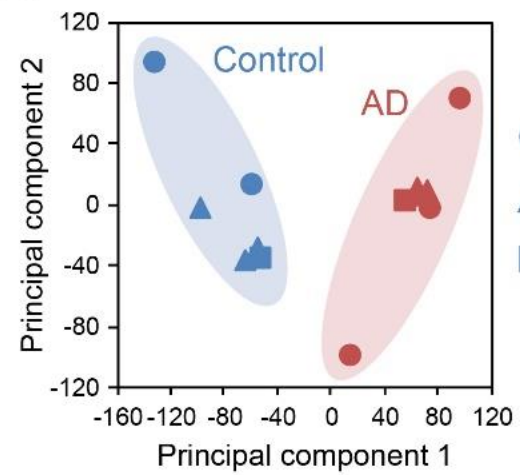

C Single cell vs 10 cells

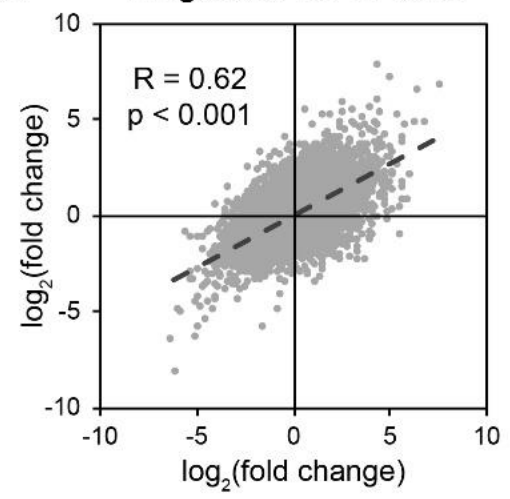

B

APP

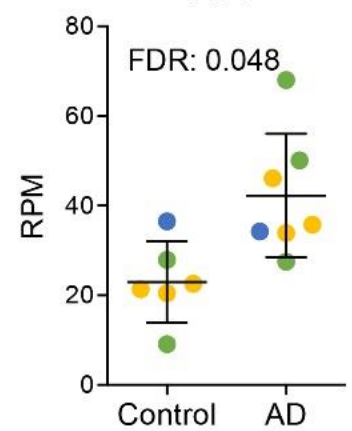

APPBP2

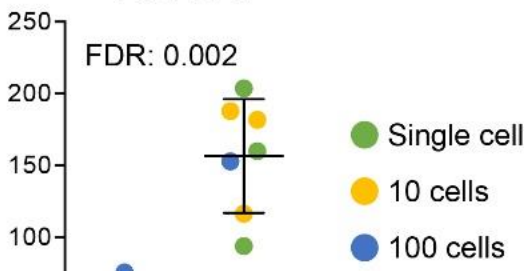

$\mathrm{D}$
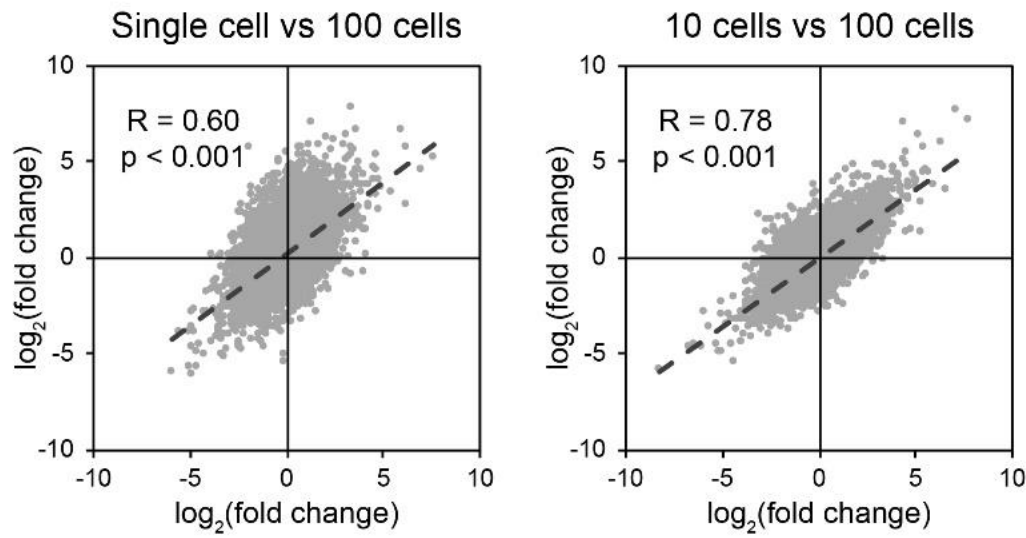

Down-regulated genes

Up-regulated genes

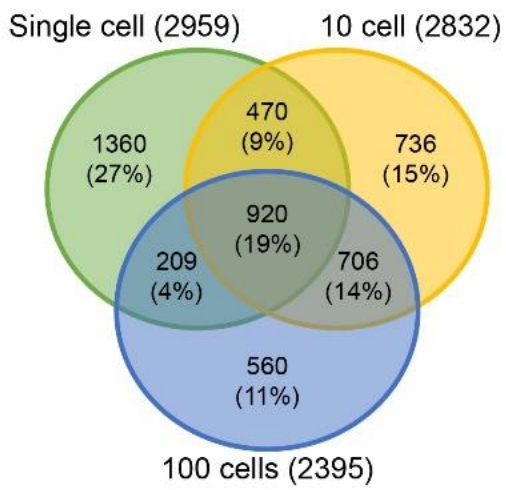

Single cell (3047) $\quad 10$ cell (2971)

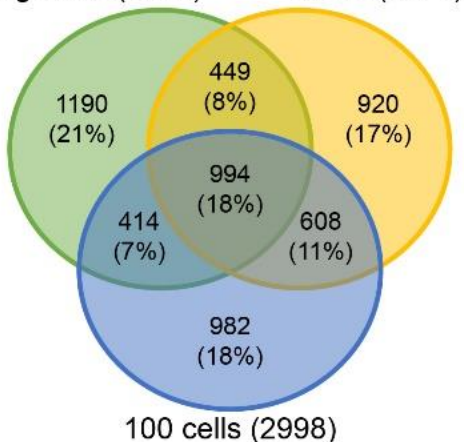

Figure 4. AmpliSeq neuron transcriptome in AD versus control. (A) Principal component analysis of gene expression between AD and control neurons. (B) The expression levels of well-characterized AD genes in AD and control neurons. (C) Correlation of gene expression changes across different neuron sets. (D) The Venn diagram of the genes with expression change in AD brain across different neuron sets. 



Figure 5. AD-related functional alterations in different neuron sets. (A) Biological functions enriched with the genes with $>2$-fold expression changes in AD brain. (B) The heatmap of genes involved in apoptosis. (C) The expression levels of wellcharacterized pro-apoptotic genes in $\mathrm{AD}$ and control neurons.

\section{Olfaction was extensively inhibited in AD hippocampus}

According to the functional analyses, olfactory system is largely reduced in the hippocampus of $\mathrm{AD}$ brain. As shown in Figure 6A, the inhibited olfactory response was mainly driven by the down-regulation of olfactory receptors. Impaired olfactory responsiveness is an early symptom of AD. Although these neurons are not directly involved in olfaction, the hippocampus does aid in olfactory memory. In our study, a total of 367 olfactory receptors were identified in hippocampus, which were expressed at moderate level in the genome of control neurons, implying the functional importance of hippocampus in olfaction (Fig. 6B). However, in AD hippocampus, the expression of the olfactory receptor family was generally suppressed (Fig. 6B). 119 family members $(32.43 \%)$ demonstrated significantly decreased expression while only 1 member $(0.27 \%)$ was increased (Fig. 6C). Of note, olfactory receptors with the highest abundance (OR11H12, OR4M1, OR4M2, OR4K2) were all significantly down-regulated in $\mathrm{AD}$ (Fig. 6B, 6D). Moreover, $\beta$-arrestin 2 (ARRB2), a crucial signal transductor of olfactory signaling, was also significantly reduced (Fig. 6A, 6D), suggesting the overall deactivation of olfactory signal transduction in AD hippocampus. 


\section{Neurotransmitter receptors were reduced in $A D$ hippocampus}

In addition to the olfactory transduction, other G-protein coupled receptor signaling pathways were also inhibited in $\mathrm{AD}$ (Fig. 7A), including neural transmission mediated by neuroactive ligand-receptor interaction. Multiple previous studies have shown inhibited neural transmission system in AD. Consistently, in our study, we found that the receptors of important neural transmission systems, including dopamine receptors (DRD1, DRD2), GABA receptors (GABRA2, GABRA5) and 5-hydroxytryamine receptors (HTR1A, HTR2A), were all significantly down regulated in $\mathrm{AD}$ neurons (Fig. 7B). It is notable that no decrements were detected for the corresponding transmitters (ligands) of these systems. Decrements in receptors for important transmission systems in the absence of corresponding decrements of transmitter synthesis suggests that $\mathrm{AD}$ therapeutic interventions targeted at increasing transmitter availability must be ineffective in the face of reduction in the ability to receive these transmitters.

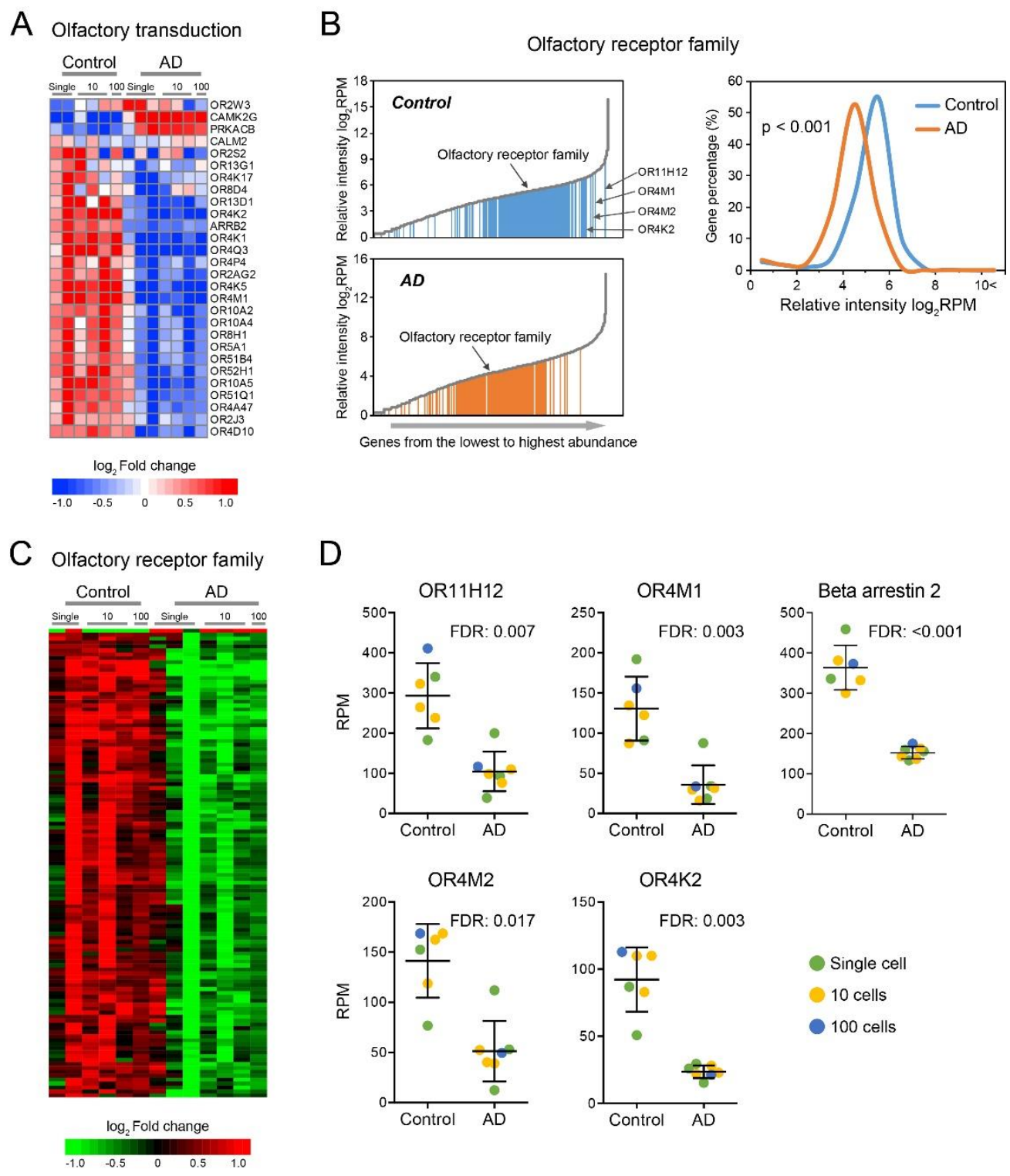

Figure 6. Olfactory transduction was significantly inhibited in AD neuron. (A) The heatmap of genes involved in olfactory signal transduction. (B) The expression distribution of olfactory receptor family in the genome of control and AD neuron. (C) The heatmap of significantly changed olfactory receptors. (D) The expression levels of the olfactory receptors with the highest abundance as well as $\beta$ arrestin 2 in $\mathrm{AD}$ and control neurons. 
A

G-protein coupled receptor signaling pathway



B

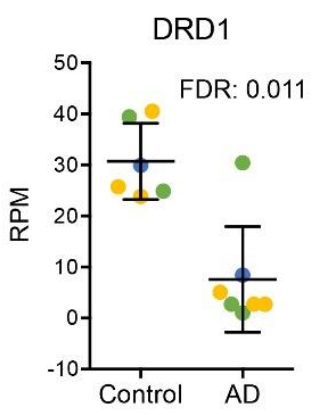

Dopamine receptors

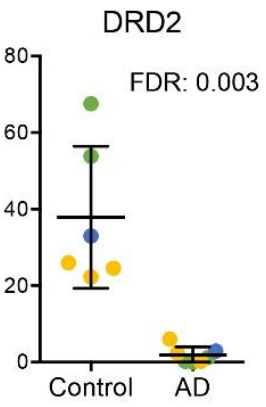

GABA receptors



GABRA5

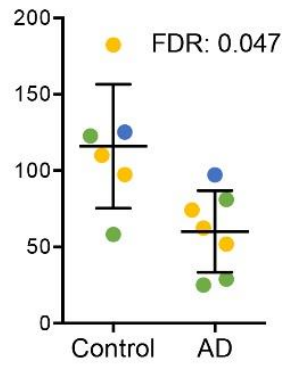

5-hydroxytryptamine receptors

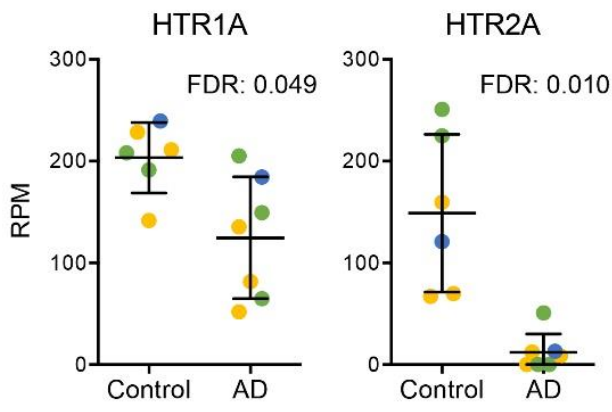

Single cell

10 cells

100 cells

Figure 7. Neurotransmitter receptors were reduced in AD neurons. (A) The functional classification of the genes involved in G-protein coupled receptor signaling pathway. (B) The expression levels of the receptors of dopamine, GABA and 5-hydroxytryptamine neurotransmission system.

\section{DISCUSSION}

The major purpose of the work described here was to determine whether the ThermoFisher Ion AmpliSeq system could be used to obtain valid data from single neurons or small sets of neurons obtained by laser capture microdissection from postmortem human brain. We profiled the gene expression in single CA1 neurons as well as sets of 10 or 100 pooled neurons that were laser captured from $\mathrm{AD}$ and control hippocampus. We found that the Ion AmpliSeq approach is robust and sensitive in measuring transcriptome at a single cell resolution. With the targeted nature of the AmpliSeq transcriptome, more than 15,000 genes were identified with very small RNA input from each single neuron, which covers a more comprehensive genome compared to the $4,000 \sim 9,000$ 
identifications per cell by other single neuron sequencing technologies [22-24]. Since laser capture microdissection limited the section thickness to $15 \mu \mathrm{m}$, smaller than that of a neural cell, only a portion of the RNA was captured from each single neuron, which further confirmed increased sensitivity of the AmpliSeq method. Moreover, the data obtained from AmpliSeq system were both internally consistent among single neurons and pooled neuron sets, as well as consistent with changes in gene expression known to typify AD neurons from affected brain regions. Known changes in $\mathrm{AD}$ are associated with alterations in cell death [30-32], metabolic activity [3336], synaptic structure and function [37-39], transmitter receptors [40], and olfaction [41, 42] - all of these responses were detected in our data from single neurons as well as pooled sets of 10 or 100 neurons.

Additionally, we found consistent reduction in $\mathrm{AD}$ hippocampal CA1 neurons of genes related to olfactory system, particularly the reduction of the olfactory receptor family. Although decrements in olfactory function in $\mathrm{AD}$ are well known [41, 42], global decrements in expression of genes related to olfaction in CA1 of hippocampus have not received much emphasis. However, there is ample evidence to associate the hippocampus with decreased olfactory function in AD. Imaging studies showed reduced hippocampal volume to be associated with decreased ability to identify odors [41] and morphological evidence documented projections from the olfactory bulb to the hippocampus [43]. Additionally, electrophysiological data have demonstrated potentials evoked in the hippocampus by stimulation of the olfactory bulb [44]. In addition to olfactory receptors, we also provided evidence that the receptors, but not the transmitters, of other neural transmission system (dopamine, GABA and 5-hydroxytryamine receptors) were also coordinately downregulated in AD hippocampal neurons. These results might have therapeutic implications; increasing the sensitivity of receptors may be more clinically effective than simply increasing the level of neurotransmitters per se.

Importantly, there are classes of transcripts that disappear as data moves from single neurons to pooled sets of neurons, and the converse - class(es) that appear as data moves from single neurons to sets of neurons. In the case of the former (glial cell development and response to oxidative stress) we assume that these are processes that happen to be present at relatively low levels in the small number of single neurons profiled, but then become swamped when more neurons are added to the mix. In the case of processes that are absent in the single neurons sampled, but increasingly present as more neurons are added to the mix (e.g. metabolic processes and synaptic transcripts) we consider these data in the context of the data on apoptotic processes and cell death in the single neurons we have selected. This suggests that the small number of single neurons selected were on the way toward cell death, and therefore, reducing expression of metabolism and synapse-related transcripts. As more neurons are added to the analysis, cell death signals decrease, metabolic processes increase, suggesting healthier neurons and synapse transcripts increase. The differences between single cell data and data from sets of 10 or 100 neurons emphasize the heterogeneity of single neuronal responses and the need for more data at the single cell level in order to truly capture the complex pathophysiology that is triggered in AD. Nevertheless, data such as those in the PCA and heat map analyses also suggest that for the present data, this heterogeneity/diversity exists within broader frameworks that may allow distinction of pertinent classes such as disease versus healthy states.

There are a few caveats. As an exploratory study, the sample size used here was limited. Although our data show internal consistency as well as the consistency with the literature, the details of the results describing differential gene expression between $\mathrm{AD}$ and control brain need to be verified in more neurons from more patients and cannot be considered as stand-alone descriptions of the $\mathrm{AD}$ brain. Using laser capture microdissection may lose information about the spatial localization of the cells selected. This loss may be partially mitigated by bar-coding cells selected for laser capture as well as by capturing more neurons across larger anatomic locales to get a more comprehensive view of AD-related neural alterations. Ultimately, further exploratory studies such as this should provide the basis for calculating power in order to pursue larger formal studies to compare single cell profiles and variations in $\mathrm{AD}$ versus normal aged brains.

\section{Conclusion}

This proof-of-concept study suggests that the Ion AmpliSeq methodology presents a useful alternative to other methods of obtaining expression data from single neurons or collections of neurons.

\section{Acknowledgements}

This study is supported by NIH grants.

\section{References}

[1] Hardy J, Selkoe DJ (2002). The amyloid hypothesis of Alzheimer's disease: progress and problems on the road to therapeutics. Science, 297:353-356.

[2] Guillozet AL, Weintraub S, Mash DC, Mesulam MM (2003). Neurofibrillary tangles, amyloid, and memory 
in aging and mild cognitive impairment. Arch Neurol, 60:729-736.

[3] Kim J, Basak JM, Holtzman DM (2009). The role of apolipoprotein E in Alzheimer's disease. Neuron, 63:287-303.

[4] Ballatore C, Lee VM, Trojanowski JQ (2007). Taumediated neurodegeneration in Alzheimer's disease and related disorders. Nat Rev Neurosci, 8:663-672.

[5] Castillo-Carranza DL, Nilson AN, Van Skike CE, Jahrling JB, Patel K, Garach P, et al. (2017). Cerebral Microvascular Accumulation of Tau Oligomers in Alzheimer's Disease and Related Tauopathies. Aging Dis, 8:257-266.

[6] Mastroeni D, Sekar S, Nolz J, Delvaux E, Lunnon K, Mill J, et al. (2017). ANK1 is up-regulated in laser captured microglia in Alzheimer's brain; the importance of addressing cellular heterogeneity. PLoS One, 12:e0177814.

[7] Lam B, Masellis M, Freedman M, Stuss DT, Black SE (2013). Clinical, imaging, and pathological heterogeneity of the Alzheimer's disease syndrome. Alzheimers Res Ther, 5:1.

[8] Badhwar A, Brown R, Stanimirovic DB, Haqqani AS, Hamel E (2017). Proteomic differences in brain vessels of Alzheimer's disease mice: Normalization by PPARgamma agonist pioglitazone. J Cereb Blood Flow Metab, 37:1120-1136.

[9] Xu Y, Liu X, Shen J, Tian W, Fang R, Li B, et al. (2018). The Whole Exome Sequencing Clarifies the Genotype- Phenotype Correlations in Patients with Early-Onset Dementia. Aging Dis, 9:696-705.

[10] Rosi S (2018). The role of peripherally derived monocytes in the aging injured brain. Cond Med, $1: 350-354$.

[11] Leak RK (2018). Conditioning Against the Pathology of Parkinson's disease. Cond Med, 1:143-162.

[12] Keren-Shaul H, Spinrad A, Weiner A, MatcovitchNatan O, Dvir-Szternfeld R, Ulland TK, et al. (2017). A Unique Microglia Type Associated with Restricting Development of Alzheimer's Disease. Cell, 169:12761290 e1217.

[13] Artegiani B, Lyubimova A, Muraro M, van Es JH, van Oudenaarden A, Clevers H (2017). A Single-Cell RNA Sequencing Study Reveals Cellular and Molecular Dynamics of the Hippocampal Neurogenic Niche. Cell Rep, 21:3271-3284.

[14] Ofengeim D, Giagtzoglou N, Huh D, Zou C, Yuan J (2017). Single-Cell RNA Sequencing: Unraveling the Brain One Cell at a Time. Trends Mol Med, 23:563576.

[15] Eberwine J, Yeh H, Miyashiro K, Cao Y, Nair S, Finnell R, et al. (1992). Analysis of gene expression in single live neurons. Proc Natl Acad Sci U S A, 89:3010-3014.

[16] Chow N, Cox C, Callahan LM, Weimer JM, Guo L, Coleman PD (1998). Expression profiles of multiple genes in single neurons of Alzheimer's disease. Proc Natl Acad Sci U S A, 95:9620-9625.

[17] Li W, Turner A, Aggarwal P, Matter A, Storvick E, Arnett DK, et al. (2015). Comprehensive evaluation of
AmpliSeq transcriptome, a novel targeted whole transcriptome RNA sequencing methodology for global gene expression analysis. BMC Genomics, 16:1069.

[18] Papp AC, Azad AK, Pietrzak M, Williams A, Handelman SK, Igo RP, Jr., et al. (2018). AmpliSeq transcriptome analysis of human alveolar and monocyte-derived macrophages over time in response to Mycobacterium tuberculosis infection. PLoS One, 13:e0198221.

[19] Birdsill AC, Walker DG, Lue L, Sue LI, Beach TG (2011). Postmortem interval effect on RNA and gene expression in human brain tissue. Cell Tissue Bank, 12:311-318.

[20] Walker DG, Whetzel AM, Serrano G, Sue LI, Lue LF, Beach TG (2016). Characterization of RNA isolated from eighteen different human tissues: results from a rapid human autopsy program. Cell Tissue Bank, 17:361-375.

[21] Huang da W, Sherman BT, Lempicki RA (2009). Systematic and integrative analysis of large gene lists using DAVID bioinformatics resources. Nat Protoc, 4:44-57.

[22] Krishnaswami SR, Grindberg RV, Novotny M, Venepally P, Lacar B, Bhutani K, et al. (2016). Using single nuclei for RNA-seq to capture the transcriptome of postmortem neurons. Nat Protoc, 11:499-524.

Nichterwitz S, Chen G, Aguila Benitez J, Yilmaz M, Storvall H, Cao M, et al. (2016). Laser capture microscopy coupled with Smart-seq2 for precise spatial transcriptomic profiling. Nat Commun, 7:12139.

[24] Lacar B, Linker SB, Jaeger BN, Krishnaswami SR, Barron JJ, Kelder MJE, et al. (2016). Nuclear RNAseq of single neurons reveals molecular signatures of activation. Nat Commun, 7:11022.

[25] Ianov L, De Both M, Chawla MK, Rani A, Kennedy AJ, Piras I, et al. (2017). Hippocampal Transcriptomic Profiles: Subfield Vulnerability to Age and Cognitive Impairment. Front Aging Neurosci, 9:383.

[26] Cembrowski MS, Wang L, Sugino K, Shields BC, Spruston N (2016). Hipposeq: a comprehensive RNAseq database of gene expression in hippocampal principal neurons. Elife, 5:e14997.

[27] Readhead B, Haure-Mirande JV, Funk CC, Richards MA, Shannon P, Haroutunian V, et al. (2018). Multiscale Analysis of Independent Alzheimer's Cohorts Finds Disruption of Molecular, Genetic, and Clinical Networks by Human Herpesvirus. Neuron, 99:64-82 e67.

[28] Zeisel A, Munoz-Manchado AB, Codeluppi S, Lonnerberg P, La Manno G, Jureus A, et al. (2015). Brain structure. Cell types in the mouse cortex and hippocampus revealed by single-cell RNA-seq. Science, 347:1138-1142.

[29] Lake BB, Ai R, Kaeser GE, Salathia NS, Yung YC, Liu $\mathrm{R}$, et al. (2016). Neuronal subtypes and diversity revealed by single-nucleus RNA sequencing of the human brain. Science, 352:1586-1590.

[30] Liang WS, Dunckley T, Beach TG, Grover A, 
Mastroeni D, Ramsey K, et al. (2008). Altered neuronal gene expression in brain regions differentially affected by Alzheimer's disease: a reference data set. Physiol Genomics, 33:240-256.

[31] Ray M, Zhang W (2010). Analysis of Alzheimer's disease severity across brain regions by topological analysis of gene co-expression networks. BMC Syst Biol, 4:136.

[32] van Rooij JGJ, Meeter LHH, Melhem S, Nijholt DAT, Wong TH, Netherlands Brain B, et al. (2019). Hippocampal transcriptome profiling combined with protein-protein interaction analysis elucidates Alzheimer's disease pathways and genes. Neurobiol Aging, 74:225-233.

[33] Mosconi L (2013). Glucose metabolism in normal aging and Alzheimer's disease: Methodological and physiological considerations for PET studies. Clin Transl Imaging, 1.

[34] Liang WS, Reiman EM, Valla J, Dunckley T, Beach TG, Grover A, et al. (2008). Alzheimer's disease is associated with reduced expression of energy metabolism genes in posterior cingulate neurons. Proc Natl Acad Sci U S A, 105:4441-4446.

[35] Meles SK, Pagani M, Arnaldi D, De Carli F, Dessi B, Morbelli S, et al. (2017). The Alzheimer's disease metabolic brain pattern in mild cognitive impairment. J Cereb Blood Flow Metab, 37:3643-3648.

[36] Herholz K, Haense C, Gerhard A, Jones M, AntonRodriguez J, Segobin S, et al. (2018). Metabolic regional and network changes in Alzheimer's disease subtypes. J Cereb Blood Flow Metab, 38:1796-1806.

[37] DeKosky ST, Scheff SW (1990). Synapse loss in frontal cortex biopsies in Alzheimer's disease: correlation with cognitive severity. Ann Neurol, 27:457-464.

[38] Selkoe DJ (2002). Alzheimer's disease is a synaptic failure. Science, 298:789-791.

[39] Coleman PD, Yao PJ (2003). Synaptic slaughter in Alzheimer's disease. Neurobiol Aging, 24:1023-1027.

[40] Xu Y, Yan J, Zhou P, Li J, Gao H, Xia Y, et al. (2012). Neurotransmitter receptors and cognitive dysfunction in Alzheimer's disease and Parkinson's disease. Prog Neurobiol, 97:1-13.

[41] Vassilaki M, Christianson TJ, Mielke MM, Geda YE, Kremers WK, Machulda MM, et al. (2017). Neuroimaging biomarkers and impaired olfaction in cognitively normal individuals. Ann Neurol, 81:871882.

[42] Yu Q, Guo P, Li D, Zuo L, Lian T, Yu S, et al. (2018). Olfactory Dysfunction and Its Relationship with Clinical Symptoms of Alzheimer Disease. Aging Dis, 9:1084-1095.

[43] White LE (1965). Olfactory bulb projections of the rat. The Anatomical Record, 152:465-480.

[44] Cragg BG (1960). Responses of the hippocampus to stimulation of the olfactory bulb and of various afferent nerves in five mammals. Exp Neurol, 2:547572. 